\title{
Calidad de vida y estado funcional de ancianos con enfermedad renal crónica estadio 5 en terapia dialítica
}

\author{
Quality of life and functional status of elderly with \\ chronic kidney disease stage 5 in dialysis therapy
}

\author{
Heidi Liliana Martínez, César Augusto Restrepo, Fernando Arango • \\ Manizales (Colombia)
}

\section{Resumen}

Objetivo: evaluar calidad de vida, estado funcional, aspectos cognitivos y afectivos en pacientes mayores de 65 años con enfermedad renal crónica estadio 5 en terapia dialítica

Material y métodos: pacientes de 65 años o más con enfermedad renal crónica estadio 5 en terapia dialítica por más de 90 días (hemodiálisis (HD ) o diálisis peritoneal (DP)), sin demencia ni reciente hospitalización. En ellos se evaluaron variables sociodemográficas y se aplicaron escalas geriátricas validadas para los siguientes aspectos: discapacidad, fragilidad, deterioro cognoscitivo, depresión y comorbilidad. También se aplicó el instrumento específico KDQOL 1.3 (versión validada en español latino de uso libre) para evaluar calidad de vida en paciente con enfermedad renal crónica en terapia dialítica.

Análisis estadístico: estudio analítico de corte transversal, descriptivo determinando distribución de frecuencias, medidas de tendencia central, medidas de dispersión; en el análisis bivariado calidad de vida y estado funcional en relación con variables: sociodemográficas, afectivas, cognitivas, fragilidad y comorbilidad y posteriormente se realizó una regresión logística múltiple.

Resultados: 90 pacientes cumplieron los criterios de inclusión, de los cuales 60 pertenecían al programa de HD y 30 a DP. La edad promedio en HD $74.1 \pm 6.1$ y en DP $72.6 \pm 6$ años. $49.55 \%$ hombres en HD y $43.7 \%$ en DP, tiempo promedio en HD $74.2 \pm 56.8$ meses, y en DP46.5 \pm 33.9 meses. El KT/V en HD fue $1.53 \pm 0.433$ y en DP $2.29 \pm 0.66 .51 \%$ de los pacientes en HD tenían criterios de depresión moderada y 5\% de depresión severa. Para el grupo de DP 56\% con depresión moderada sin criterios para depresión severa. Se encontró fragilidad en un $68 \%$ para el grupo de HD y $53.3 \%$ para el grupo de DP. 56\% de pacientes del grupo de HD eran completamente independientes y $40 \%$ en el grupo de DP. En cuanto a calidad de vida con el uso del KDQOL 1.3 los dominios con deterioro en la calidad de vida según los resultados emanados de este estudio fueron: carga de enfermedad renal crónica, síntomas de enfermedad renal, trabajo, función física y dolor. Los determinantes de mejor calidad de vida por dominios fueron: ser hombre, estar en diálisis peritoneal, tener buen nivel de independencia medido por la escala de Barthel y la edad (a menor edad mejor calidad de vida). Mientras que los determinantes de peor calidad de vida fueron: ser mujer, tener síntomas depresivos (según tamizaje con escala Yesavage) tener compromiso en el ABC físico con algún grado de dependencia física según el Barthel, pertenecer al grupo de hemodiálisis, y la edad (a mayor edad peor calidad de vida).

Conclusiones: en general el estado funcional de este grupo de pacientes muestra un adecuado nivel de independencia. En cuanto a la calidad de vida se encuentra que los dominios más afectados para el grupo analizado son dolor, función física, síntomas de enfermedad renal y carga de enfermedad renal, al igual que trabajo, los cuales se relacionan con algunos predictores intervenibles como síntomas depresivos y tipo de diálisis, esto hace presumir que la intervención de dichas variables tendrían implicaciones directas en la calidad de vida de este grupo poblacional. (Acta Med Colomb 2015; 40: 13-19).

Palabras clave: insuficiencia renal crónica, diálisis, calidad de vida, anciano.
Dra. Heidi Liliana Martínez Sánchez: Residente de Medicina Interna y Geriatría, Universidad de Caldas; Dr. César Augusto Restrepo Valencia: Internista Nefrólogo, Profesor Asociado Universidad de Caldas. Dr. Fernando Arango: Pediatra, Neonatólogo, Epidemiólogo, Universidad de Caldas, Universidad del Valle. Manizales (Colombia).

Correspondencia: Dr. César Augusto Restrepo Valencia. Manizales (Colombia). E-mail: caugustorv@une.net.co Recibido: 17/III/2014 Aceptado: 23/II/2015 


\begin{abstract}
Objective: to evaluate quality of life, functional status, cognitive and affective aspects in patients over 65 with stage 5 chronic kidney disease on dialysis therapy.

Patients, materials and methods: patients 65 years and older with chronic kidney disease stage 5 in dialysis therapy for more than 90 days (hemodialysis (HD ) or peritoneal dialysis (PD)) without dementia or recent hospitalization. Sociodemographic variables were evaluated and geriatric validated scales for the following aspects were applied: disability, frailty, cognitive impairment, depression and comorbidity. The specific instrument 1.3 KDQOL for assessing quality of life in patients with chronic kidney disease on dialysis therapy was also applied.
\end{abstract}

Statistical analysis: analytical cross-sectional descriptive study, determining frequency distributions, measures of central tendency, measures of dispersion; in the bivariate analysis, quality of life and functional status in relation to variables: sociodemographic, affective, cognitive, frailty and comorbidity and a multiple logistic regression was subsequently performed.

Results: 90 patients met the inclusion criteria, of which 60 belonged to the program HD and 30 to PD. Mean age in HD was $74.1 \pm 6.1$ and in PD $72.6 \pm 6$ years. $49.55 \%$ were men in HD and $43.7 \%$ in DP; average time on HD $74.2 \pm 56.8$ months and $46.5 \pm 33.9$ months in PD. KT / V in HD was $1.53 \pm 0.433$ and $2.29 \pm 0.66 \mathrm{i}$ PD. $51 \%$ of HD patients met criteria for moderate depression and $5 \%$ for severe depression. For the group of PD, $56 \%$ with moderate depression without criteria for major depression. Fragility was found in 68\% for the HD group and 53.3\% for the group of PD. $56 \%$ of patients in the HD group were completely independent and $40 \%$ in the group of PD. In terms of quality of life with the use of KDQOL 1.3 domains with deterioration in quality of life according to the results from this study were: burden of chronic kidney disease, symptoms of kidney disease, work, physical function and pain. Determinants for better quality of life domains were: being male, being on peritoneal dialysis, have a good level of independence measured by the Barthel scale and age (the younger, the better quality of life), while the determinants of worse quality of life were: being female, having depressive symptoms (as screening with Yesavage scale), have involvement in physical ABC with some degree of physical dependence according to Barthel, belong to the group of hemodialysis, and age ( the older, worse quality of life).

Conclusions: in general the functional status of these patients shows an appropriate level of independence. As for the quality of life, the most affected domains for the analyzed group are pain, physical function, symptoms of kidney disease and renal disease burden, as well as work, which relate to some predictors on intervention such as depressive symptoms and type of dialysis; this presumes that the intervention of such variables would have direct implications on the quality of life for this population group. (Acta Med Colomb 2015; 40: 13-19).

Keywords: chronic renal failure, dialysis, quality of life, elderly,

\section{Introducción}

La enfermedad renal crónica (ERC) grado 5 (G5) (tasa de filtración glomerular inferior a $15 \mathrm{~mL} / \mathrm{min} / 1.73 \mathrm{~m}^{2} \mathrm{sc}$ ) es una patología con implicaciones médicas importantes, dadas las múltiples funciones renales: regulación del equilibrio electrolítico y ácidobase, excreción de productos nitrogenados de desecho, control de la presión arterial, maduración de eritrocitos, síntesis de la forma activa de vitamina D y participación en la gluconeogénesis. La ERC expone crónicamente al paciente a alteraciones bioquímicas e inflamatorias que impactan diferentes sistemas de manera nociva. En ancianos la ERC es una patología que favorece el desarrollo de discapacidad por sí misma dado el aumento de interleucinas proinflamatorias, alteraciones nutricionales, osteoarticulares, hematológicas, mentales, entre otras derivadas de la enfermedad, que propenden al desarrollo de fragilidad y a su vez de la posibilidad de discapacitarse (1-3).

Dentro de los modelos de atención al anciano con enfermedad renal crónica G5, se ha discutido a lo largo de diferentes estudios las diversas clases de terapias (incluida la diálisis) que se aplican en cuanto al impacto en sobrevida $(4,5)$, pero indiscutiblemente el estado funcional y la calidad de vida cobran fuerza, entendiendo que cada intervención se dimensiona en la medida que se alcancen los propósitos terapéuticos indicados con el menor efecto adverso posible $(6,7)$.

La idea de este estudio fue evaluar la calidad de vida, estado funcional, aspectos cognitivos y afectivos en pacientes $\geq 65$ años con enfermedad renal crónica G5 en terapia de reemplazo renal en una unidad renal en la ciudad de Manizales 


\section{Material y métodos}

Pacientes de 65 años o más cumplidos, con diagnóstico de enfermedad renal crónica G5, quienes requerían terapia de diálisis crónica (hemodiálisis o diálisis peritoneal), tratados en una unidad renal de la ciudad de Manizales (CaldasColombia-Sur América) durante el periodo de recolección de datos que tuvo lugar en el mes de septiembre de 2013. Los pacientes debían de llevar al menos 90 días en terapia dialítica, sin haber cambiado su modalidad en los últimos 60 días. Se excluyeron pacientes con diagnóstico de demencia, y aquellos que no firmaron el consentimiento informado.

En los pacientes se evaluaron variables sociodemográficas, seguridad social, estrato socioeconómico, estado civil, institucionalización, calidad de vida, estado funcional, comorbilidades, depresión, aspectos psicológicos, sociales, cognitivos y fragilidad. La adecuación de la terapia dialítica se evaluó mediante el KT/V, considerándose para los pacientes en hemodiálisis un valor satisfactorio una cifra mayor a 1.2 y para diálisis peritoneal un valor mayor a la cifra 1.6.

Para valorar el tamizaje cognitivo (deterioro cognitivo) se aplicó el Minimental Test, para depresión la escala de depresión geriátrica de Yesavage (GSD). El grado de independencia por las escalas de valoración funcional (capacidad funcional (ABC FISICO): Índice de Barthel $\mathrm{y}$ (capacidad funcional (ABC instrumental)) Escala de Lawton modificada. La valoración de fragilidad según la escala de fragilidad validada en español ENASEM. Calidad de vida con el uso del KDQOL-SF versión 1.3 en español.

El estudio fue aprobado por el colectivo del programa de medicina interna-geriatría y Comité de Bioética de la Universidad de Caldas, también por el comité de ética local, comité científico nacional y director científico nacional de la empresa prestadora del servicio de diálisis en la cual estaban siendo tratados los pacientes. Se trató de un estudio analítico de corte transversal. Para el análisis estadístico se realizó una base de datos con el programa EXCEL, los cuales fueron procesados por medio del software Stata 12, se utilizó estadística descriptiva: frecuencias, medidas de tendencia central y de dispersión. En cuanto a las asociaciones de deterioro funcional y calidad de vida con las diferentes características sociodemográficas, afectivas, cognitivas, fragilidad y comorbilidad, se realizó un análisis bivariado y se calcularon las razones de prevalencia. Además se construyeron modelos de regresión logística univariada con las variables que tenían valor de $\mathrm{P}<0.20$. A continuación, se realizó un modelo de regresión logística múltiple con las variables que presentaron valor de $\mathrm{P}<0.20$. Los resultados se presentaron como OR y sus respectivos intervalos de confianza de $95 \%$.

\section{Resultados}

El tiempo de ejecución del estudio fue de 18 meses. Se revisó la base de datos de la institución y se encontró un total de 120 ancianos con enfermedad renal crónica G5, 72 se encontraban en el brazo de hemodiálisis y 48 en el brazo de diálisis peritoneal. Treinta pacientes fueron excluidos por no cumplir los criterios de inclusión.

La edad promedio para el grupo de hemodiálisis fue de $74.1 \pm 6.1$ y para el grupo de diálisis peritoneal $72.6 \pm 6.49$ años, la distribución por género encontró que los hombres representaban $55 \%$ del brazo de hemodiálisis y $43.7 \%$ del grupo de diálisis peritoneal. El tiempo de permanencia en diálisis para el grupo de hemodiálisis fue de 74.2 \pm 56.8 , y en el grupo de diálisis peritoneal $46.5 \pm 33.9$ meses. El KT/V en hemodiálisis fue $1.53 \pm 0.433$, y en diálisis peritoneal $2.29 \pm 0.66$. Las variables institucionalización, estrato, tipo de aseguramiento, cohabitación y dependencia económica pueden ser consultadas en la Tabla 1.

Tabla 1. Características sociodemográficas de los ancianos con enfermedad renal crónica estadio 5. Clínica Renal RTS - Manizales.

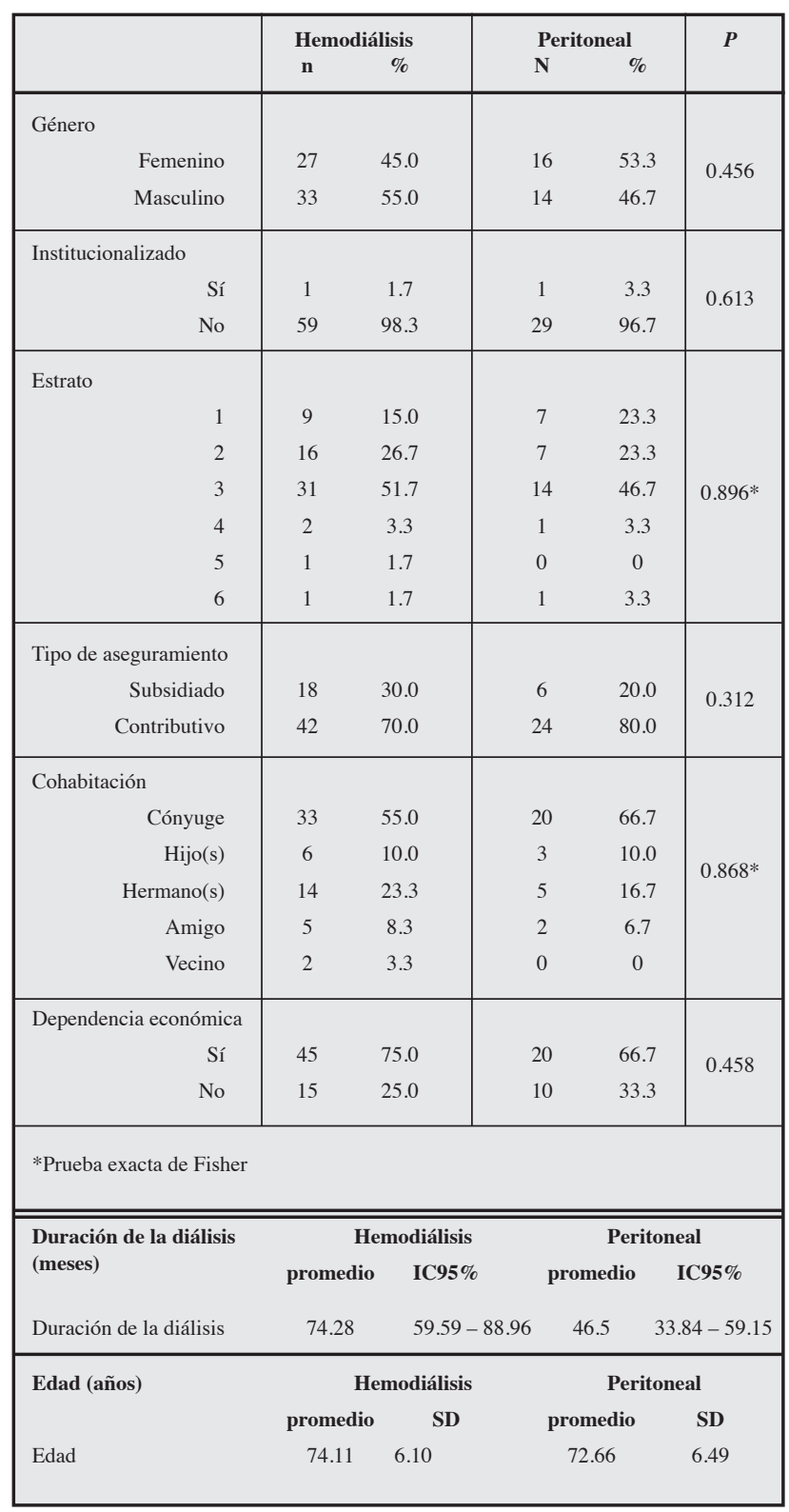


En cuanto al análisis de comorbilidad $81.7 \%$ de los pacientes en hemodiálisis padecían hipertensión y $78 \%$ de pacientes en diálisis peritoneal, el siguiente diagnóstico en frecuencia fue diabetes mellitus tipo 2, las otras variables de comorbilidad se analizan en la Tabla 2.

En el tamizaje cognitivo se encuentra un mini mental promedio de 25 puntos para ambos grupos lo cual se traduce en ausencia de deterioro cognitivo significativo para el nivel de escolaridad (Tabla 3).

Tabla 2. Comorbilidades de los ancianos con enfermedad renal crónica estadio 5. Clínica Renal RTS - Manizales.

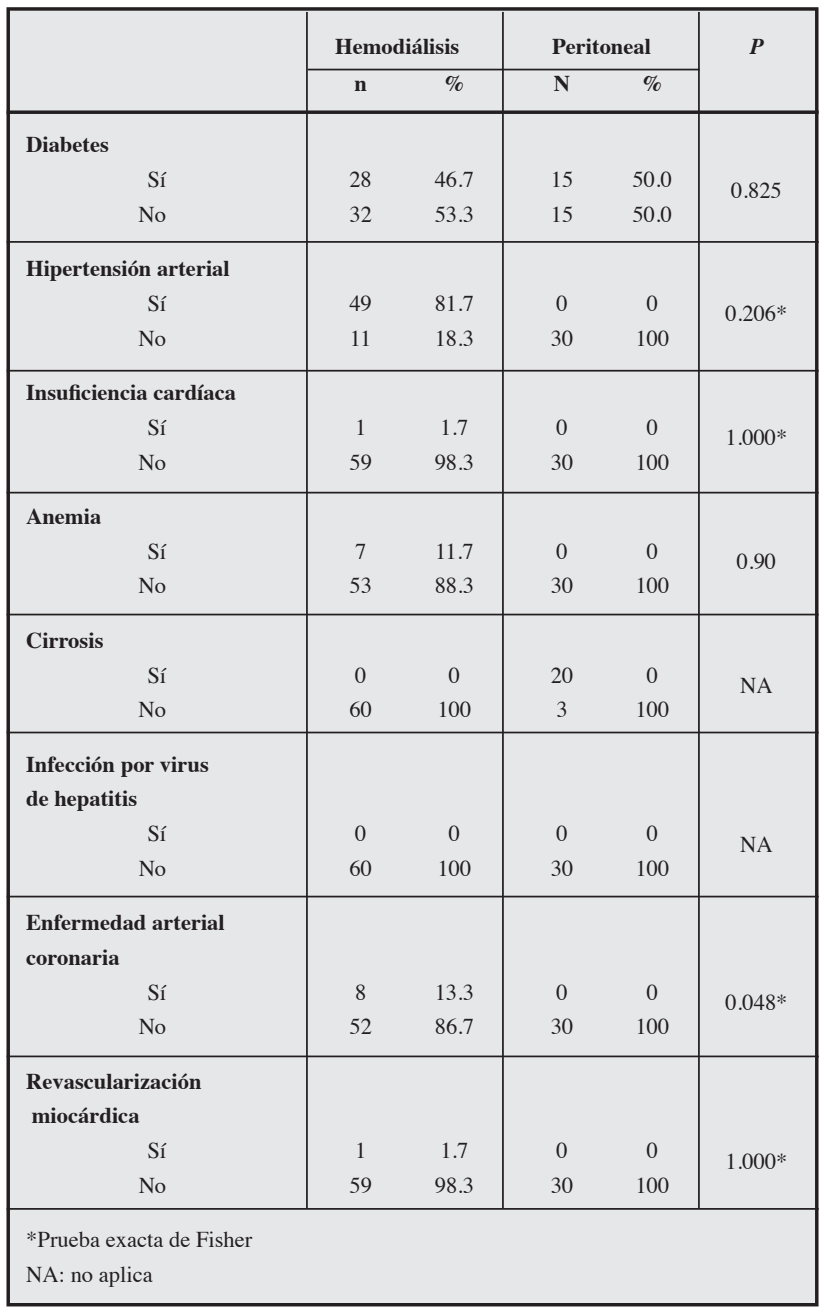

Tabla 3. Evaluación de depresión según la escala de Yesavage en los ancianos con enfermedad renal crónica estadio 5. Clínica Renal RTS - Manizales.

\begin{tabular}{|c|cc|cc|c|}
\hline & \multicolumn{2}{|c|}{ Hemodiálisis } & \multicolumn{2}{c|}{ Peritoneal } & \multirow{2}{*}{$\boldsymbol{P}$} \\
\cline { 2 - 5 } & $\mathbf{n}$ & $\mathbf{\%}$ & $\mathbf{N}$ & $\mathbf{\%}$ & \\
\hline Depresión & & & & & \\
No deprimido & 26 & 43.3 & 13 & 43.3 & \multirow{2}{*}{.662* } \\
Depresión moderada & 31 & 51.7 & 17 & 56.7 & \multirow{2}{*}{ Depresión severa } \\
Drueba exacta de Fisher & 3 & 5.0 & 0 & 0 & \\
\hline
\end{tabular}

En cuanto al tamizaje de síntomas depresivos con la escala de Yesavage mostró que $51 \%$ de los pacientes en hemodiálisis tenían criterios de depresión moderada y $56 \%$ para el grupo de peritoneal, y sólo 5\% de los pacientes con hemodiálisis mostraron cifras sugestivas de depresión severa sin diferencias estadísticamente significativas (Tabla 4).

El promedio de la escala de Barthel mostró que para el grupo de hemodiálisis $78 \%$ de los pacientes eran completamente independientes y $76 \%$ del grupo de diálisis peritoneal, la escala de Lawton arrojó que $56 \%$ de los pacientes del grupo de hemodiálisis eran completamente independientes y $40 \%$ para el grupo de diálisis peritoneal sin diferencias estadísticamente significativa (Tablas 5 y 6).

En la valoración subjetiva de fragilidad con la escala ENASEM se encontró que $68 \%$ de los pacientes con hemodiálisis eran frágiles, y $26 \%$ pre frágiles, mientras que para el grupo de diálisis peritoneal $53 \%$ eran frágiles y $46 \%$ pre

Tabla 4. Evaluación de fragilidad según criterios subjetivos en los ancianos con enfermedad renal crónica estadío 5. Clínica Renal RTS - Manizales.

\begin{tabular}{|c|c|c|c|c|c|}
\hline & \multicolumn{2}{|c|}{ Hemodiálisis } & \multicolumn{2}{|c|}{ Peritoneal } & \multirow[t]{2}{*}{$P$} \\
\hline & $\mathrm{n}$ & $\%$ & $\mathrm{~N}$ & $\%$ & \\
\hline \multicolumn{6}{|l|}{ Fragilidad } \\
\hline No frágil & 3 & 5.0 & 0 & 0 & \\
\hline Prefrágil & 16 & 26.7 & 14 & 46.7 & $0.11^{*}$ \\
\hline Frágil & 41 & 68.3 & 16 & 53.3 & \\
\hline
\end{tabular}

Tabla 5. Evaluación de capacidad funcional según el índice de Barthel en los ancianos con enfermedad renal crónica estadio 5. Clínica Renal RTS - Manizales.

\begin{tabular}{|c|c|c|c|c|c|}
\hline \multirow[b]{3}{*}{ Capacidad funcional } & \multicolumn{2}{|c|}{ Hemodiálisis } & \multicolumn{3}{|c|}{ Peritoneal } \\
\hline & promedio & IC95\% & & dio & IC95\% \\
\hline & 96.08 & $92.67-99.48$ & & & $98.03-99.63$ \\
\hline & \multicolumn{2}{|c|}{ Hemodiálisis } & \multicolumn{2}{|c|}{ Peritoneal } & \multirow[t]{2}{*}{$P$} \\
\hline & $\mathbf{n}$ & $\%$ & $\mathbf{N}$ & $\%$ & \\
\hline \multicolumn{6}{|l|}{ Capacidad funcional } \\
\hline Independiente & 47 & 78.3 & 23 & 76. & \multirow{4}{*}{$0.903 *$} \\
\hline Dependencia leve & 11 & 18.3 & 7 & 23 & \\
\hline Dependencia moderada & 1 & 1.7 & 0 & 0 & \\
\hline Dependencia severa & 1 & 1.7 & 0 & 0 & \\
\hline
\end{tabular}

Tabla 6. Evaluación de capacidad funcional instrumental según la escala de Lawton en los ancianos con enfermedad renal crónica estadío 5. Clínica Renal RTS - Manizales.

\begin{tabular}{|c|cc|cc|c|}
\hline & \multicolumn{2}{|c|}{ Hemodiálisis } & \multicolumn{2}{|c|}{ Peritoneal } & \multirow{2}{*}{$\boldsymbol{P}$} \\
\cline { 2 - 5 } & $\mathbf{n}$ & $\mathbf{N}$ & $\mathbf{N}$ & $\%$ & \\
\hline Capacidad funcional & & & & & \\
Totalmente independiente & 32 & 53.3 & 12 & 40.0 & \multirow{2}{*}{$0.667 *$} \\
Totalmente dependiente & 1 & 1.7 & 0 & 0 & \\
\hline *Prueba exacta de Fisher
\end{tabular}


frágiles sin diferencias estadísticamente significativas entre ambos grupos (Tabla 7).

En relación a la calidad de vida con el uso del KDQOL 1.3 Se consideró que los dominios con niveles $<50 \%$ estaban relacionados con una pobre calidad de vida, de los cuales los dominios con mayor compromiso según los resultados emanados de este estudio son carga de enfermedad renal crónica, síntomas de enfermedad renal, trabajo, función física y dolor. Al realizar la regresión logística se relacionan a continuación las variables con significancia estadística (Tablas 8, 9).

Se encuentra que los pacientes en la modalidad de diálisis peritoneal tienen mejor calidad vida en el dominio de síntomas de enfermedad renal, con un OR de 0.16 y un IC de 95\% 0.04-0.07 con una $\mathrm{P}<0.05$.

Se encontró además que la presencia de síntomas depresivos se asoció con un aumento de 5.8 veces del riesgo de deteriorar la calidad de vida en el dominio de carga de enfermedad renal, con IC de 95\% de 1.67-15.9 con una $\mathrm{P}<0.05$ para ambos grupos de pacientes sin importar el tipo de diálisis.

En el dominio relacionado con situación laboral o trabajo, se encontró que los pacientes en diálisis peritoneal conservan mejor calidad de vida que los pacientes en la modalidad de hemodiálisis con un OR 0.24 con IC $95 \%$ entre $0.07-0.76$ con $\mathrm{P}<0.05$.

Al evaluar el dominio función física se documenta que el género masculino disfruta de una mejor calidad de vida en comparación con el género femenino; por el contrario, los pacientes que tienen algún grado de dependencia física según lo encontrado al aplicar la escala de Barthel, tienen peor calidad de vida en el dominio función física, sin importar el tipo de diálisis al que estén adscritos, con un OR: 7.5 y con IC95\% 1.41-37 con una $\mathrm{P}<0.05$.

En lo referente al dominio dolor, se evidencia que el grupo de pacientes que se encuentra en la modalidad de
Tabla 7. Evaluación de deterioro cognitivo según el minimental test en los ancianos con enfermedad renal crónica estadío 5. Clínica Renal RTS - Manizales.

\begin{tabular}{|l|cc|cc|}
\hline \multirow{2}{*}{} & \multicolumn{2}{|c|}{ Hemodiálisis } & \multicolumn{2}{c|}{ Peritoneal } \\
\cline { 2 - 5 } & promedio $\quad$ IC95\% & promedio & IC95\% \\
\hline Deterioro cognitivo & 25.23 & $24.14-26.31$ & 25 & $23.45-26.54$ \\
\hline
\end{tabular}

Tabla 8. Evaluación de la calidad de vida según KDQOL-SF en los ancianos con enfermedad renal crónica estadío 5. Clínica Renal RTS - Manizales.

\begin{tabular}{|l|c|c|}
\hline Variable & $\begin{array}{c}\text { Hemodiálisis } \\
\text { mediana }\end{array}$ & $\begin{array}{c}\text { Peritoneal } \\
\text { mediana }\end{array}$ \\
\hline Lista de síntomas/problemas & 87.50 & 82.29 \\
Efectos de enfermedad renal & 56.25 & 81.25 \\
Carga de enfermedad renal & 37.50 & 43.75 \\
Situación laboral & 0.0 & 0.0 \\
Función cognitiva & 73.33 & 90.0 \\
Calidad de interacción social & 66.67 & 66.67 \\
Función sexual & 100 & 77.50 \\
Sueño & 72.50 & 66.67 \\
Soporte social & 83.33 & 100 \\
Incentivo por el equipo de diálisis & 100 & 83.33 \\
Satisfacción del paciente & 83.33 & 42.50 \\
Funcionamiento físico & 45.0 & 12.50 \\
Función física & 0.0 & 80.0 \\
Dolor & 77.50 & 50.0 \\
Salud general & 50.0 & 60.0 \\
Bienestar emocional & 72.0 & 62.50 \\
Función emocional & 0.0 & 55.0 \\
Función social & 62.50 & \\
Energía/fatiga & & \\
\hline
\end{tabular}

Tabla 9. Modelo final de regresión logística múltiple.

\begin{tabular}{|c|c|c|c|c|}
\hline & OR crudo & IC95\% & OR ajustado* & IC95\% \\
\hline $\begin{array}{l}\text { Síntomas de enfermedad renal } \\
\text { Diálisis peritoneal }\end{array}$ & 0.27 & $0.04-1.02$ & 0.16 & $0.04-0.67 * *$ \\
\hline Carga de enfermedad & 1.78 & $0.84-3.80$ & 5.15 & $1.67-15.9^{* *}$ \\
\hline $\begin{array}{r}\text { Diálisis peritoneal } \\
\text { Estado civil divorciado }\end{array}$ & $\begin{array}{l}0.73 \\
0.52\end{array}$ & $\begin{array}{l}0.34-1.52 \\
0.08-2.40\end{array}$ & $\begin{array}{l}0.24 \\
0.18\end{array}$ & $\begin{array}{c}0.07-0.76^{* *} \\
0.03-1.03\end{array}$ \\
\hline $\begin{array}{l}\text { Función física } \\
\text { Género masculino } \\
\text { Algún grado de dependencia } \\
\text { Depresión }\end{array}$ & $\begin{array}{c}0.50 \\
2 \\
1.85\end{array}$ & $\begin{array}{l}0.22-1.09 \\
0.83-4.74 \\
0.83-4.26\end{array}$ & $\begin{array}{l}0.26 \\
7.25 \\
2.46\end{array}$ & $\begin{array}{c}0.09-0.70 * * \\
1.41-37.36 * * \\
0.91-6.59\end{array}$ \\
\hline Diálisis peritoneal & 2 & $0.48-8.13$ & 8.56 & $1.22-59.97 * *$ \\
\hline
\end{tabular}


Figura 1. Flujograma.

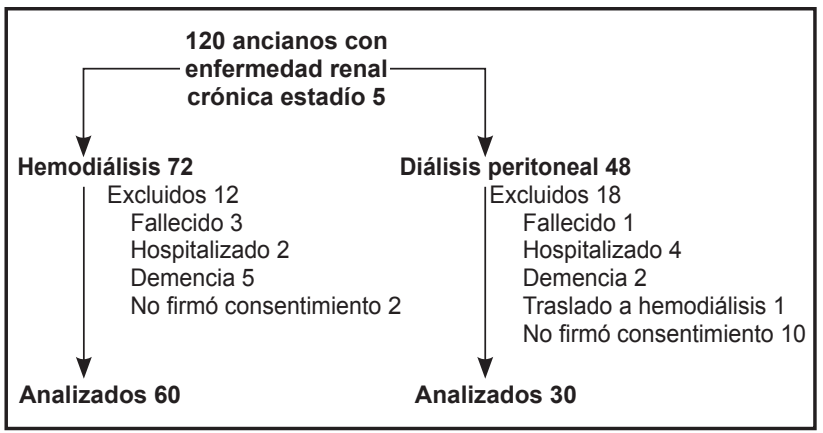

diálisis peritoneal se deteriora hasta 8.5 veces la calidad de vida relacionada con síntomas dolorosos en comparación con la modalidad hemodiálisis, con un IC 1.22-57 y P $<0.05$.

\section{Discusión}

El clínico que se enfrenta día a día al anciano con enfermedad renal crónica G5, tiene que tomar la decisión de iniciar o no la terapia de reemplazo renal. Aunque parece ser una determinación fácil en términos conceptuales desde la medicina interna, realmente no lo es en términos de realidad e individualidad, es allí donde el médico internista-geriatra desempeña un papel fundamental debido a que el anciano debe ser visto desde la óptica multidimensional, para determinar la opción indicada en lo referente a sobrevida, pero a su vez en cuanto a calidad de vida y discapacidad $(6,7)$, estos últimos determinados según la pertinencia de las diferentes intervenciones médicas, que tengan en cuenta una óptica equilibrada.

Lo anterior justifica la valoración geriátrica multidimensional en la cual la calidad de vida juega un papel importante al envejecer. El concepto calidad de vida se ajusta a consideraciones multidimensionales como autopercepción de salud, acceso a servicios de salud, niveles de satisfacción y muchos otros dominios que deberán ser considerados detalladamente.

Las mediciones en calidad de vida en pacientes con terapia dialítica revelan conclusiones controversiales debido al uso de diferentes instrumentos que aun en algunos casos pueden no ser pertinentes (8).

La multiplicidad de instrumentos disponibles con este fin genera una divergencia en las conclusiones, lo cual no permite tener una aproximación universal del impacto de la terapia dialítica en la calidad de vida de las poblaciones geriátricas $(8,9)$, por el contrario en lo que sí existe consenso es que las intervenciones en otros factores asociados modificables como anemia, fragilidad, rehabilitación física previa y aplicación de los principios básicos de cuidado paliativo han mostrado impacto positivo en la medición de la calidad de vida de este grupo de pacientes $(10,11)$.

Noshad y cols (12) compararon los desenlaces de calidad de vida en pacientes con enfermedad renal crónica en terapia de hemodiálisis o diálisis peritoneal, y aplicaron la escala
GHQ 28, encontrando cuatro dominios específicos muy deteriorados: disfunción psicosocial, alteración del sueño, síntomas depresivos y estrés, demostrando en su estudio que la diálisis peritoneal a diferencia de la hemodiálisis ofrecía una mejor calidad de vida para los dominios reportados.

Ruiz y cols (13), en su estudio de valoración de calidad de vida de pacientes ancianos con diálisis en España, demostraron que el dominio reacción emocional como análogo a depresión se correlacionaba con peor calidad de vida, medida y comparada con varios instrumentos entre los cuales estaban el SF 12 y el KDQOL; concomitante los dominios sueño, función física, dolor y movilidad, fueron los dominios más afectados para este grupo poblacional, resultados similares a los detectados en nuestro estudio, encontrándose como predictores la edad, funcionalidad (medido con Karnofsky) y el índice de comorbilidad de Charlson.

En nuestro estudio, se documenta un buen nivel de independencia para ambos grupos analizados según tipo de diálisis y tiempo promedio en diálisis. En estudios previos se ha observado que la declinación funcional para este grupo poblacional se presenta especialmente en los primeros tres a seis meses posteriores al inicio de la terapia de remplazo renal (14), tiempo ampliamente superado por ambos grupos de pacientes en nuestra población.

Con respecto a los resultados de calidad de vida encontramos cinco dominios predominantemente afectados: síntomas de enfermedad renal, carga de enfermedad renal, trabajo, dolor y función física; es de resaltar que en este estudio se demuestra que el tipo de diálisis a la cual los pacientes son sometidos es un predictor independiente y estadísticamente significativo, especialmente para dos dominios específicos de la calidad de vida: síntomas de enfermedad renal y situación laboral, siendo la diálisis peritoneal el predictor de mejor calidad de vida en comparación con los pacientes sometidos a hemodiálisis.

Los predictores de buena calidad de vida en cuanto a la función física que muestra este estudio es el género y la edad, entendiéndose que a menor edad mejor función física, sin embargo y pese a explorar otros aspectos reportados en la literatura (15), como la presencia de enfermedad arterial coronaria, enfermedad cerebrovascular, enfermedad arterial periférica, éstos no fueron concluyentes dada la limitación en el tamaño de la muestra; no obstante, otro determinante reportado en la literatura para función física es el nivel sérico de albúmina (6), el cual no se evaluó en este estudio por lo cual este ítem no es comparable con otros autores.

Según el tamizaje para depresión realizado en este estudio se presenta una asociación directa con el dominio carga de enfermedad y síntomas depresivos con tendencia a empeorar la calidad de vida en este dominio siendo estadísticamente significativo; a su vez los síntomas depresivos muestran una tendencia a afectar negativamente el dominio de función física; sin embargo, este último no se pudo demostrar con significancia estadística, probablemente en función del tamaño de muestra. 
Desde el punto de vista de la literatura regional, la información disponible en este aspecto y para este grupo poblacional específico es limitada, por lo cual la discusión de este estudio se basa en la información disponible mas no comparable en todos los casos para el grupo etario específico; además hay que considerar que es necesario realizar más estudios que revelen los determinantes concretos en relación con la calidad de vida de este grupo, y que permitan generar herramientas operativas para la toma de decisiones desde el punto de vista de intervención clínica, con el fin de favorecer los desenlaces a todo nivel.

\section{Limitaciones}

Se considera que las principales limitaciones de este estudio son: el tamaño de la muestra, lo que dificulta el análisis de algunas variables que mostraron una tendencia, pero que no alcanzaron significancia estadística, especialmente para el grupo de diálisis peritoneal, por lo cual no se puede considerar que estos hallazgos expliquen el grosor de esta población a nivel regional e inclusive nacional. No incluimos en nuestro estudio los medicamentos ni dosis que recibían los pacientes, variable que impacta sin lugar a dudas la adherencia y calidad de vida de los ancianos, y que bien podría ser motivo para un posterior estudio.

\section{Conclusiones}

En general el estado funcional de este grupo de pacientes muestra un adecuado nivel de independencia. En cuanto a la calidad de vida se encuentra que los dominios más afectados para el grupo analizado son dolor, función física, síntomas de enfermedad renal y carga de enfermedad, al igual que trabajo; en relación con los predictores ya analizados en la regresión logística (Tabla 9), no obstante en la gran mayoría de estudios se incluye la fragilidad como un predictor independiente para calidad de vida como para funcionalidad (2), no se pudo demostrar en este estudio probablemente por limitación en el tamaño de la muestra.

\section{Conflicto de interés}

Los autores realizaron el trabajo con fines de tipo meramente académico y sin ningún conflicto de interés.

\section{Fuentes de apoyo}

Ninguna

\section{Agradecimientos}

Dr. Carlos Arturo Hoyos: Internista Geriatra Docente Universidad de Caldas. Dr Yajaira Carrasco: Psicóloga Clínica Unidad Renal RTS de Caldas. Sr. Fabián Torres: Estudiante Licenciatura en Biología y Química.

\section{Referencias}

1. Jefrey B. Halter, Joseph Ouslander, Mary Tinetti, Stephanie Studensky, Kevin P High, Hazzard"S. Geriatric Medicine and Gerontology, Sixth Edition, McGrawHill 2009 Parte IV, section D, paginas 1009-1045.

2. Guevara Farfán G M, Restrepo CA, Buitrago CA, Torres J, Serna J. El riñón en el anciano. En Nefrología Básica 2, Editorial La Patria 2012, pag 441-448, Manizales.

3. Brown EA, Johansson L. Epidemiology and management of end-stage renal disease in the elderly. Nat Rev Nephrol 2011; 7: 591-598.

4. Odenn MC. Physical Functioning in elderly Persons With Kidney Disease. Adv Chronic Kidney Dis 2010; 17: 348-357.

5. Hallan SI, Dahl K, Oien CM, Grootendorst DC, Aasberg A, Holmen J, Dekker FW. Screening strategies for chronic kidney disease in the general population: follow-up of cross sectional health survey. BMJ 2006; 333: 1047.

6. Bowling CB, O'Hare AM. Managing older adults with CKD: individualized versus disease-basedapproaches. Am J Kid Dis 2012; 59: 293-302.

7. Demoulin N, Beguin C, Labriola L, Jadout M. Preparing renal replacementtherapy in stage 4 CKD patients referred tone phrologists: a difficult balance between futility and insufficiency. A cohort study of 386 patients followed in Brussels. Nephrol Dial Transplant 2011; 26: 220-226.

8. Jassal SV, Chiu E, Hladunewich M. Loss of independence in patients starting dialysis at 80 years of age or older. N Engl J Med 2009; 361: 1612-1613.

9. Rakowski DA, Caillard S, Agodoa LW, Abbot KC. Dementia as a predictor of mortality in dialysis patients. Clin J Am Soc Nephrology 2006; 1: 1000-1005.

10. Cook WL. The Intersection of geriatrics and Chronic Kidney Disease: Frailty and Disability Among Older Adults with kidney disease. Adv Chronic Kidney Dis 2009; 16: 420-429

11. Murtagh FE, Marsh JE, Donohoe P, Ekbal NJ, Sheerin NS, Harris FE. Dialysis or not? A comparative survival study of patients over 75 years with chronic kidney disease stage 5. Nephrol Dial Transplant 2007; 22: 1955-1962.

12. Chandna SM, Da Silva-Gane M, Marshall C, Warwicker P, Greenwood RN, Farrington K. Survival of elderly patients with stage 5 CKD: comparison of conservative management and renal replacement therapy. Nephrol Dial Transplan 2011; 26: 1608-1614.

13. Murtagh FE, Addington-Hall JM, Higginson IJ. End-stage renal disease: a new trajectory of functional decline in the last year of life. J Am Geriatr Soc 2011; 59: 304-308.

14. Carson RC, Juszczak M, Davenport A, Burns A. Is maximum conservative management an equivalent treatment option o dialysis for elderly patients with significant comorbid disease? Clin J Am Soc Nephro 2009; 4: 1611-1619.

15. Ledebo I, Ronco $\mathbf{C}$. The best dialysis therapy? Results from an international survey among nephrology professionals. NDT Plus 2008; 1: 403-408. 